\title{
Allomorphy —its logic and limitations: opening remarks from the guest editors
}

\author{
Noam Faust ${ }^{1} \cdot$ Nicola Lampitelli ${ }^{2}$
}

Received: 22 March 2016 / Accepted: 29 March 2016 / Published online: 6 April 2016

CC Springer Science+Business Media Dordrecht 2016

\section{Introduction}

Allomorphy is the phenomenon whereby the same grammatical information is conveyed in two or more forms in complementary distribution. Because the conditions that produce allomorphy may be of many different natures, allomorphy occupies a central role in linguistic theory, and has considerable consequences for our conception of Language, how it is constructed and represented, how it stored and what its computation might be sensitive to. It is with such questions in mind that we organized the conference "Allomorphy: its logic and limitations", held at the Hebrew University of Jerusalem in July 2014. ${ }^{1}$ This special volume of Morphology holds ten of the papers presented in that conference. In this short introduction, we will review the overarching questions raised in the conference, present the papers in the volume and summarize the findings and the predictions arrived at in the volume.

\footnotetext{
${ }^{1}$ The conference was made possible thanks to a grant from the Israeli Science Foundation (Grant no. 0322917). Additional funding was provided by the Faculty of Humanities in the Hebrew University and by the Language, Logic and Cognition Center of the university. The organizer of the conference (the first editor above) would especially like to thank Nora Boneh, Edit Doron, Danny Fox, Malka Hovav and Ivy Sichel for their encouragement and help. Much of the editing was conducted when the affiliation of Noam Faust was to the Hebrew University of Jerusalem.
}

\footnotetext{
$\varangle$ N. Faust

noam.faust@univ-paris8.fr

N. Lampitelli

nicola.lampitelli@univ-tours.fr

1 Université Paris 8, CNRS UMR 7023, Paris, France

2 LLL, Université François Rabelais de Tours \& CNRS, Tours, France
} 


\section{What is allomorphy?}

The first and most basic question that was raised in the conference was: what are the conditions that must hold for a phenomenon to be called allomorphy? Many researchers adopt the view that allomorphy must involve more than one lexical representation. Under this view, the existence of two realizations, say [t] and [d] or [ə] and [ən], for the same grammatical information, is not enough in order to define the phenomenon as allomorphic; Phonology could be managing the realization of a single underlying representation, in which case at the level of the knowledge of the speaker, there is no allomorphy. In other words, in order to apply the term allomorphy to a certain distribution, one must show that the different realizations do not somehow follow from the general phonological rules of the language as applied to a single representation. Interestingly, this places allomorphy on a different level than its phonological counterpart, allophony: whereas allophony involves only one specific piece of information (the phoneme) to which general rules are applied, the one-to-many relation in allomorphy does not follow from general rules and must be memorized.

All that said, cases that can be viewed as simple phonology are often found in textbooks as canonical examples of allomorphy. This is because what one researcher may allow herself to posit as a unifying lexical representation for a purported allomorphic situation might seem too abstract to another, who might prefer a solution with two underlying representations. It is not always possible to judge in favor of one view over the other on the basis of anything other than elegance.

Three papers in this volume relate to these issues. The first paper, written by Renate Raffelsiefen, has the empirical basis of vowel quality in German. The paper further refines the notion of allomorphy by indicating the relevance of Output-Output ("OO") relations. It is shown that such relations are evaluated on the phonemic level, rather than the phonetic level. Real allomorphy is then defined as everything that violates OO-correspondence, i.e. everything that involves distinctness at the phonemic level. Since subphonemic variance does not violate OO-correspondence like allomorphy does, they are expected to function differently in the linguistic system. Indeed, Raffelsiefen shows that there is a pressure for real allomorphy to disappear (for instance in obsc[i]n - obsc[i]nity), whereas for the subphonemic variance of allophony there is no such pressure.

The second paper, written by Eva Zimmermann, proposes a "monorepresentational" analysis of purported cases of allomorphy. Instead of allowing two distinct lexical representations to co-exist, Zimmermann's account posits a single underlying form and derives surface alternations in the phonology through the use of OT and coloured containment theory. The paper is especially interesting since it treats the little-studied phenomenon of apparent allomorphy in tone realization, in two casestudies from little-known languages. San Miguel el Grande Mixtec (Otomanguean, Amuzgo-Mixtecan) exhibits positional variation in the realization of a morpheme (= the same phonological item is realized in two distinct positions); and Kalam Kohistani (Indo-Aryan, Dardic) realizes the same morphological category with two distinct tones ( $\mathrm{H}$ and $\mathrm{L}$ ). In both cases, it is claimed, the choice of the locus and quality of the realization follow from general and language-specific phonological principles, given a single underlying form. The point is made again - although this time for very 
different types of data-that if there is only one lexical representation, there is no allomorphy.

The paper by Tobias Scheer, which will be discussed below, also presents the monorepresentational argument. The notable difference between the two papers is the component which must be enriched in order to accommodate the single representation: Scheer's analysis enriches mainly the representation, while Zimmermann's also adduces fine-tuned constraints.

\section{What are the grammatical limits on allomorphy?}

It is a hallmark of generative linguistics to account not only for the attested, but also for the unattested. The latter effort is expressed in the study of allomorphy by asking: what are the grammatical limits on allomorphy? How does the architecture of grammar constrain the identity of trigger and target of allomorphy, and how does it constraint what trigger may influence what target? May languages differ in the types of allomorphy they allow, and if so, why, and what correlates with such differences?

Four papers in the present volume discuss such limitations on allomorphy. The first is by Beata Moskal and Peter Smith. The authors first show that a theory of allomorphy containing both cyclic and linear locality is falsified by phenomena from in several languages. They propose a theory without linear locality; such a theory incorporates hyper-contextual Vocabulary Insertion-rules. These rules "make reference to more than one node, which jointly form the context for VI." Locality, often assumed to be a condition imposed on allomorphy by the grammar, is epiphenomenal under this view.

The second paper to discuss the issue of grammatical limitations on allomorphy is by Amy-Rose Deal. Deal examines the distribution of plural marking in Nez Perce (Penutian), whose distribution has an intriguing pattern. Whereas both nouns and adjectives may be overtly marked for plurality, if the referent is inanimate, only the adjectival modifier takes the plural affix. In her account, Deal shows that this can be accounted for by assuming that "the conditioning of allomorphy must favor nonphonological factors over phonological ones". Another pattern examined is the double plural marking on reduplicated adjectives. This pattern, it is argued, is best understood as a two-stage process. First, the positions of the reduplicant are inserted, and then the phonology is responsible for the association of segmental material to these positions.

The paper by Tobias Scheer examines the typology of allomorphy. It reveals a curious generalization, namely that allomorphy is never conditioned by melody (i.e. specific segments, as opposed to "consonant" or "vowel"). The paper shows that this limitation follows from a modular view of language, specifically from the claim that one module cannot directly interpret the "vocabulary" of another module, but it can parse the output of that module. Thus, morphology cannot be sensitive to segments, but it can be sensitive to sonority or syllabic (or skeletal) positions, because these are the outputs of phonological calculation. It is further shown that non-modular, globalist theories that intertwine phonology and morphology cannot account for this generalization equally well. As mentioned above, like Zimmermann, Scheer also proposes single underliers for many cases of purported allomorphy. Special importance 
is attributed to representations with floating segments in this discussion. Finally, this single-underlier approach is compared to accounts with two competing underliers; it is shown that the latter overgenerates again, specifically when allomorph selection is not optimizing in any way.

The last paper on the limits of allomorphy is by Noam Faust. It targets the traditional distinction between strong and weak suppletions, which have repeatedly been claimed to be essentially the same. The empirical basis of the paper is root allomorphy as manifested in the defective roots of Semitic. Evidence is brought forth in favor of distinguishing between three levels of representation of roots: the formless root, the phonological index, and the exponent or underlying representation. At each of these levels, allomorphy takes a different guise and has a different effect. Allomorphy of the formless root is strong suppletion; allomorphy of the phonological index is weak suppletion, not phonologically-optimizing, but possibly lexicallyoptimizing (for instance, in unifying paradigms); and allomorphy of the exponent is phonologically-optimizing. Interestingly, since Semitic roots have internal structure, allomorphy always target a discrete part of the root; a central result of the paper is thus that strong suppletion aside, there is in fact never any root allomorphy in Semitic.

To summarize, the papers in this part of the volume show how several proposed universal primitives of grammars-modules, phases, spell-out, levels of representation-influence the effects, the nature, and the mere possibility of allomorphy in natural language.

\section{What may trigger allomorphy?}

Another overarching topic of the conference was allomorph selection and the different considerations influencing it. What, besides immediately adjacent phonological structure, can favor the selection of one allomorph over the other? In other words, what rationales can be found for allomorph selection other than the familiar ones? The last four papers in this volume belong to this direction of exploration.

Daniel Asherov \& Outi Bat-El treat the notions of "default" and "elsewhere," in the expression of gender on nominal bases in Modern Hebrew. They show that there is a local default and a global default; only the latter is a real "elsewhere" allomorph. The local default, while still acting as default, is more specific to a certain morphosyntactic context, while the global default will hold for that context and elsewhere only when the local default is blocked by phonological considerations. Thus, the paper makes a case for such phonological considerations influencing the selection of one default or the other. Finally, an interesting case is examined where both affixes are attested; speakers are tested with wug words and behave against the predictions from frequency, but with the predictions of the proposal.

The paper by Evan Gary-Cohen \& Lior Laks, also based on data from Modern Hebrew, examines allomorphs in free distribution. In this language, participles may be used either as verbs or as adjectives. For passive participles, if the second consonant of the stem is historically a guttural, the preceding vowel may surface as $[\mathrm{u}]$ or [o]. An experiment shows that the distribution is not entirely free: in their verbal use, the forms will surface more with [u] than with [o], whereas in the adjectival use it 
is the other way around. The authors relate this finding to the claim that passive verbs are derived in the syntax, while adjectives are derived in the lexicon: allomorph selection is therefore influenced by the component in which the complex word is derived. Gradability follows from additional influences, such as the presence of comparable paradigms the lexicon, but not in the syntax.

In their study, Mohamed Lahrouchi and Rachid Ridouane claim that templatic considerations allow one to understand allomorph selection. Moroccan Arabic double plurals (broken and sound) are analyzed as instantiations of two distinct syntactic positions, a low and a high one. The former is not only the locus of broken plural, but also of diminutivization. This configuration reflects the observation that broken plurals and diminutives are in complementary distribution. Indeed, diminutives form their plural exclusively by suffixation. The last part of the paper is devoted to an acoustic study of emphasis spread: it is shown that emphasis is blocked by cyclicity of the syntactic derivation. Its limit corresponds precisely to the positional distinction between broken and sound plurals.

Finally, Radwa Fathi and Jean Lowenstamm analyze the many realizations of gender in French nouns. They propose a novel analysis of noun structures which accounts also for the well-known case of floating consonants. The major aim of this work is to shape the borders between arbitrariness vs. systematicity in gender selection in French. Assuming both adjunction and probing, Fathi and Lowenstamm identify four distinct syntactic configurations. Each configuration underlies a particular sub-group of nouns: two-gendered nouns with a floating consonant, two-gendered nouns under adjunction, one-gendered nouns with no floating consonants and onegendered nouns with a floating final consonant.

These last four papers illustrate the diverse rationales that may lead to allomorphy, thereby making the case for its centrality in the study of the workings of language in general.

\section{Predictions}

As a summary of the import of the present volume and in the interest of future studies, we would like to emphasize one prediction made in each paper. As can be expected from a multi-authored volume, the predictions may be slightly incompatible with respect to each other. Also note that the choice of the prediction to highlight is oursthe specific predictions are not necessarily discussed at length in the paper itself.

1. Allomorphy is cognitively costly because it involves violations of output-output correspondences; there will therefore be pressure to eliminate allomorphy, but not allophony.

2. Complex phonological computation entails simpler lexical representations and thus a single underlier.

3. Hyper-contextual VI-rules are preferable to the adjacency condition, because they reduce suppletion to a case of general allomorphy.

4. The melodic content of reduplicants cannot be present at vocabulary insertion; therefore, it cannot trigger allomorphy. Non-phonological conditioning takes precedence over phonological conditioning. 
5. Allomorphy should not be triggered by phonological primes, such as melody; this must hold whether the allomorphy is phonologically-optimizing or not.

6. Strong and weak suppletion are not the same process and apply to different levels of representation; they will show different effects (for instance with respect to optimization).

7. The default allomorph might not be the elsewhere allomorph.

8. The component of grammar in which a word is derived and the accessibility of analogous paradigms influence the choice of allomorphs.

9. Allomorphy may result from certain grammatical markers competing for the same template position; in this case, syntactic locality will determine realization.

10. Two-gender languages will enforce regularities about gender exponence exclusively when pairs are involved.

We invite the readers of this volume to examine the detailed motivations behind these findings and predictions, which we hope will prove to be significant for future research. 